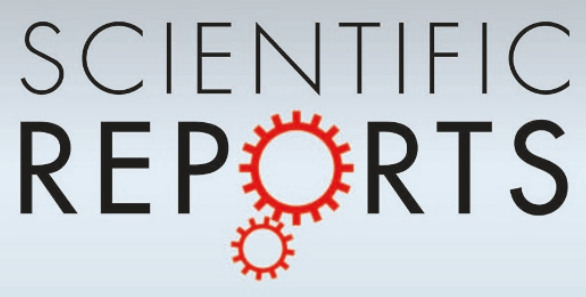

OPEN

SUBJECT AREAS:

NANOPARTICLES

APPLIED OPTICS

ELECTRICAL AND ELECTRONIC

ENGINEERING

SOLAR CELLS

Received

28 March 2013

Accepted

20 June 2013

Published

9 July 2013

Correspondence and requests for materials should be addressed to

E.H.S. (ted.sargent@ utoronto.ca)

* These authors contributed equally to this work.

\section{Folded-Light-Path Colloidal Quantum Dot Solar Cells}

\author{
Ghada I. Koleilat*, Illan J. Kramer*, Chris T. O. Wong, Susanna M. Thon, André J. Labelle, \\ Sjoerd Hoogland \& Edward H. Sargent
}

Department of Electrical and Computer Engineering, University of Toronto, 10 King's College Road, Toronto, Ontario M5S 3G4, Canada.

Colloidal quantum dot photovoltaics combine low-cost solution processing with quantum size-effect tuning to match absorption to the solar spectrum. Rapid advances have led to certified solar power conversion efficiencies of over 7\%. Nevertheless, these devices remain held back by a compromise in the choice of quantum dot film thickness, balancing on the one hand the need to maximize photon absorption, mandating a thicker film, and, on the other, the need for efficient carrier extraction, a consideration that limits film thickness. Here we report an architecture that breaks this compromise by folding the path of light propagating in the colloidal quantum dot solid. Using this method, we achieve a substantial increase in short-circuit current, ultimately leading to improved power conversion efficiency.

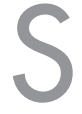
olution-processed solar cells offer the promise of low cost, large-area processing, and, prospectively, high solar power conversion efficiencies ${ }^{1-4}$. Colloidal quantum dots (CQDs) have attracted interest as photovoltaic light harvesters because, in addition to being processed from the solution phase, their bandgap can be tuned via synthetic control over nanoparticle size to optimize their spectral properties in the absorption of sunlight ${ }^{5-8}$.

Unfortunately, standing in the way of higher solar power efficiency is the fact that CQD materials today exhibit limited transport lengths ${ }^{9-11}$ compared to traditional, high-temperature-processed bulk semiconductors.

There exists therefore an urgent need to enhance absorption in a given thickness of CQD solid. Optical enhancements of absorption in ultrathin film semiconductor photovoltaics offer avenues to overcoming limited electronic transport in these materials. Progress has recently been made in this direction by engineering both the geometric ${ }^{12-15}$ and electronic landscapes ${ }^{16}$ in CQD devices, as well by improving the electrical back contacts at the semiconductor/metal interface ${ }^{17,18}$. Plasmonic absorption enhancements were recently shown ${ }^{19}$, and multijunction architectures offer a broadband approach to increasing solar cell performance ${ }^{20,21}$.

\section{Results}

In the present work, we sought to increase the interaction of light with the absorbing CQD medium by passing light through the CQD layer multiple times. Multi-pass optical management shemes have been shown to be effective in crystalline silicon ${ }^{22-24}$ and dye-sensitized solar cells ${ }^{25}$. We would fold the light path in order to propagate light through the active layer more than the typical two normal-incidence passes. Figure 1a shows a flat depleted heterojunction ${ }^{3}$ device taking advantage of only two passes of light while a folded-light-path (FLP) device (Figure $1 \mathrm{~b}$ ) can take advantage of 6 passes, effectively absorbing all photons having energy greater than the CQD bandgap. Figure 1c illustrates the geometric constraints to achieving a periodically repeating structure. Equations relating angles $\theta_{1}, \theta_{2}$ and lengths $x_{1}, x_{2}, x_{3}, A, A_{\text {tilt }}, L, t$ and $t_{\text {tilt }}$ can be found in SI 1 .

We sought to assess quantitatively the extent to which the FLP strategy could enhance absorption, hence current. We began by exploring the spectral behaviour in the case of a $45^{\circ}$ tilt angle. We show in Figure 2 a the percentage contribution of each sequential pass in both the flat and the FLP architectures. Whereas only first and second passes can be considered in the flat case (grey and black dashed lines, respectively), we considered up to six full passes in the FLP case, wherein the length of each pass is scaled up by a factor of $1 / \sin \theta_{2}$.

The contribution of the additional passes through the CQD layer translates into added photon current through spectral multiplication with the AM 1.5 solar photon fluence spectrum ${ }^{26}$ (Figure $2 \mathrm{~b}$, blue region versus orange region). We then proceeded to explore the impact of different choices of tilt angle. Figure $2 \mathrm{c}$ compares the two pass and six pass total achievable current as a function of tilting angle. The optimal calculated angle is approximately $50^{\circ}$, yielding a net potential short-circuit current density $\left(J_{S C}\right)$ enhancement of $3 \mathrm{~mA} \cdot \mathrm{cm}^{-2}$. 
a
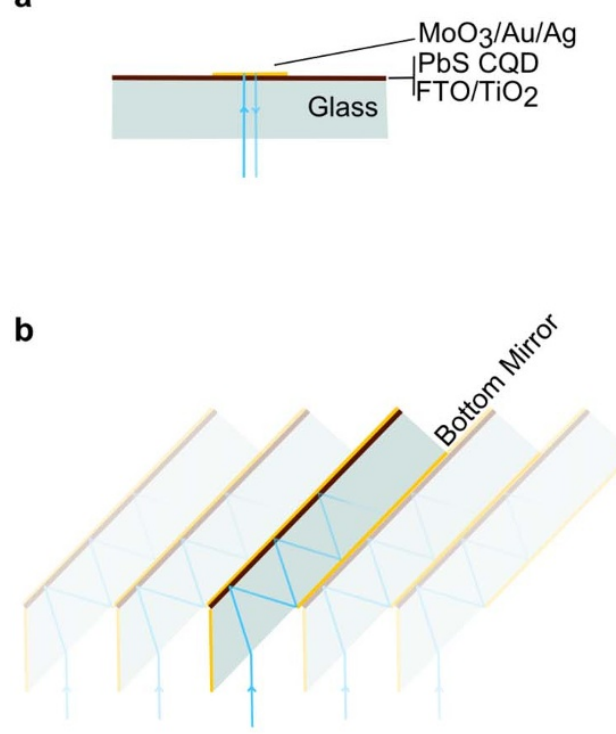

C

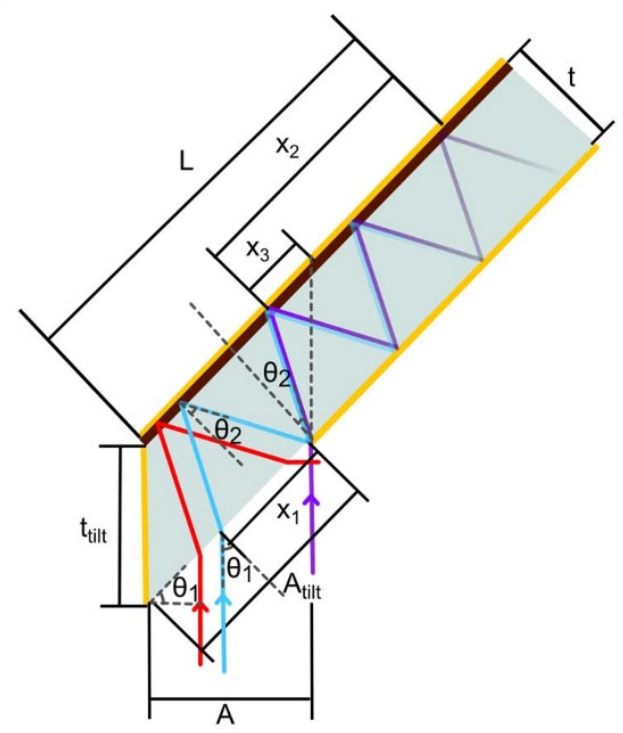

Figure 1 Configuration and geometric considerations of FLP CQD solar cells. (a) Conventional double-pass CQD solar cell. (b) Periodic arrangement of FLP CQD solar cell which takes advantage of multiple passes. (c) Geometric considerations for periodicity and multipass absorption. More details can be found in section SI 1.

We fabricated devices where the CQD materials were synthesized and applied using the highest-photovoltaic-performance previouslypublished chemistry and materials processing ${ }^{8}$. For both the conventional flat device and the FLP device, the electron-accepting $\mathrm{TiO}_{2}$ layer was deposited by RC magnetron sputtering ${ }^{27}$. Masks were developed to accommodate the evaporation of the various metallic mirrors and electronic contacts (SI 2) for the FLP device. Briefly, edge mirrors of titanium, gold and silver were deposited, followed by titania on the fluorine-doped tin oxide (FTO) plane. Following an aqueous titanium chloride treatment and thermal annealing, we deposited a back mirror consisting of gold and silver. The CQD layer was then deposited in a layer-by-layer fashion using a solid state mercaptopropionic acid (MPA) treatment. Finally an elongated top contact/mirror consisting of molybdenum trioxide, gold and silver was deposited. Controls employed conventional contacts as described previously ${ }^{8}$. For more information, see methods.

The absorption characteristics of the flat and FLP devices were then explored. First, to look for qualitative evidence of multipass, we provide in Figure 3b (inset) a photograph of a sample illuminated through its aperture at a $45^{\circ}$ tilt, leading to internal reflections and visual indication of at least six total passes through the film. This picture shows passes 1, 3 and 5 seen through the top of the device. Note that the active area of the device is defined by the light aperture, not the contact area ${ }^{28}$.

The absorption spectra of Figure $3 \mathrm{a}$ include both the total device absorption - including the glass, FTO and $\mathrm{TiO}_{2}$ layers - for the normal and $45^{\circ}$ angled case; and also the absorption contribution of the CQD film alone. The measured absorbance includes effects from both the desired quantum dot film absorption, and also the undesired substrate and electrode absorption. The external quantum efficiency ( $E Q E$, Figure $3 \mathrm{~b}$ ) of the device, on the other hand, features only the benefits of the increase in quantum dot film absorption. In the infrared spectral range, where double-pass absorption is the lowest, the measured FLP case improvement is the greatest, and the enhanced absorption and $E Q E$ follow one another most closely. In the visible spectral range, the benefits of the FLP are accrued in fact in the first pass (i.e. it is not multipass/folding, but only tilting, that benefits $E Q E$ ): absorption occurs closer to the front charge-separating interface, leading not to higher absorption, but to higher internal quantum efficiency in the tilted case. Convolving these $E Q E$ curves with the AM 1.5 solar spectrum and integrating generates expected
$J_{S C}$ values of $25 \mathrm{~mA} \cdot \mathrm{cm}^{-2}$ to $21 \mathrm{~mA} \cdot \mathrm{cm}^{-2}$ in the FLP and flat devices, respectively.

To evaluate the impact on solar power conversion efficiency, we now consider as well the implications on voltage of the FLP architecture. Collecting current from the multipass device necessitates an increase in electrode area per sun-facing area of solar cell. Thus, in the equation for open-circuit voltage $\left(V_{O C}\right)$ as a function of shortcircuit current $\left(I_{S C}\right)$, dark saturation current $\left(I_{0}\right)$ and Temperature $(T)^{29}$

$$
V_{O C}=\frac{k T}{q} \ln \left(\frac{I_{S C}}{I_{0}}+1\right)
$$

were $k$ is the Boltzmann constant and $q$ is the elementary charge, $I_{0}$ is increased by the area of the FLP electrode.

In our best FLP cells, our typical contact strip area was over 7 times larger than the aperture area due to geometric considerations, yielding an expected drop in $V_{O C}$ from our typical flat control of $0.61 \mathrm{~V}$ to $0.56 \mathrm{~V}$ (i.e. a loss of $k T / q \times \ln 7$ as predicted in equation 1 ). The $15 \%$ expected improvement in $J_{S C}$ exceeds this $8 \%$ loss in $V_{O C}$.

Experimentally, the FLP devices exhibited an enhanced current compared to planar controls of $24.6 \mathrm{~mA} \cdot \mathrm{cm}^{-2}$ versus $20.2 \mathrm{~mA} \cdot \mathrm{cm}^{-2}$ (Figure $4 \mathrm{a}, \mathrm{c}$ ), an increase of $4.4 \mathrm{~mA} \cdot \mathrm{cm}^{-2}$. The decrease in voltage (green compared to red markers in Figure $4 \mathrm{~b}$ ) also followed the predicted decrease (Figure $4 \mathrm{e}$ ) from $0.61 \mathrm{~V}$ to $0.56 \mathrm{~V}$. Overall, the power conversion efficiency, $\eta$, reported in Figure $4 \mathrm{a}$ and $\mathrm{d}$, improved to AM1.5 solar $\eta$ of 7.8 (the fill factor differences are negligible as they fall within the measurement error). For reference, Figure $4 \mathrm{f}$ and g illustrate the expected gain in current and $\eta$ as a function of FLP electrode pixel strip length when considering purely optical light capture. Clearly notable is the 2 pass regime under $\sim 3.25 \mathrm{~mm}$, the 4 pass regime from $\sim 3.25 \mathrm{~mm}$ to $\sim 6 \mathrm{~mm}$ and the 6 pass regime from $\sim 6 \mathrm{~mm}$ to $\sim 8.75 \mathrm{~mm}$. Subsequent passes were not considered as they contribute very little to additional $J_{S C}$.

\section{Discussion}

It is evident that any up-front absorption by the substrate and transparent contacts would compound unfavourably in a multipass context. To quantify this, we turned to the same model employed in Figure 2 while accounting for the glass, FTO and $\mathrm{TiO}_{2}$ layers of our typical depleted heterojunction devices. The electron-accepting 

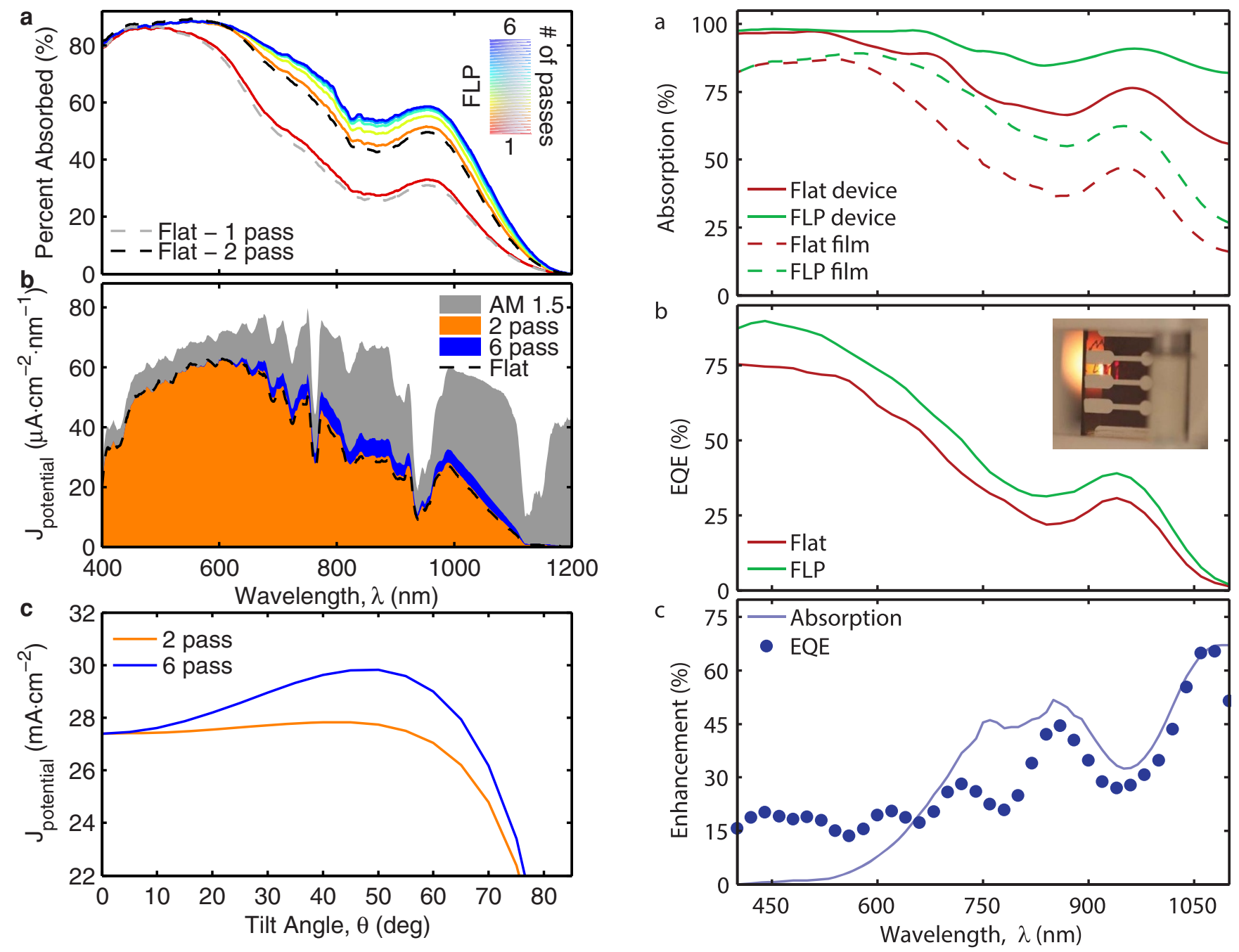

Figure $2 \mid$ Theoretical contributions of multiple passes on $J_{S C}$.

(a) Absorption contribution of the first two passes (grey and black dashed lines) of a flat DH CQD device and of the first six passes (colored lines) of a FLP DH CQD device. (b) Spectral current contribution of the first two passes and subsequent four passes of a FLP DH CQD device. The two-pass flat contribution is shown as dashed black curve. Grey area represents the AM 1.5 spectrum. (c) Tilting angle impact on total potential output current considering two passes (orange curve) and six passes (blue curve).

$\mathrm{TiO}_{2}$ is $\sim 500 \mathrm{~nm}$ thick and is formed from a colloidal paste. Figure $5 \mathrm{a}, \mathrm{d}$ and g-left highlight the contribution of each of these layers, highlighting the impact of each in a $45^{\circ}$ tilted FLP context. The thick $\mathrm{TiO}_{2}$ layer alone absorbs the photon equivalent of $6 \mathrm{~mA} \cdot \mathrm{cm}^{-2}$ of short-circuit current (red bar, Figure $5 \mathrm{~g}$ ), leaving the CQD layer with a best-case $26 \mathrm{~mA} \cdot \mathrm{cm}^{-2} J_{S C}$ (yellow bar, Figure $5 \mathrm{~g}$ ). By employing a thinner, sputtered $\mathrm{TiO}_{2}$ layer (Figure 5b, e and g-middle), the FLP device improves its best-case $J_{S C}$ to $30 \mathrm{~mA} \cdot \mathrm{cm}^{-2}$. This is still far from the theoretical ideal $1.3 \mathrm{eV}$ bandgap maximum of $36 \mathrm{~mA} \cdot \mathrm{cm}^{-230}$. To further illustrate the importance of all layers in the FLP stack, we also modeled the best-case scenarios for all layers in the stack, including thinner glass, highly transparent indium tin oxide (ITO), sputtered $\mathrm{TiO}_{2}$ as well as a perfect reflector at the top contact over all wavelengths of interest (Figure $5 c$, $f$ and g-right). In this case, only Fresnel reflections at the front surface of the cell and the absorption coefficients of the CQD layer itself limit absorption. Through this analysis, it is possible to achieve over $34 \mathrm{~mA} \cdot \mathrm{cm}^{-2}$ of $J_{S C}$.

This third condition, wherein the active layer has access to nearly all incident photons, suggests a further avenue to optimizing a solar cell employing a folded light path. The incoupling and trapping of

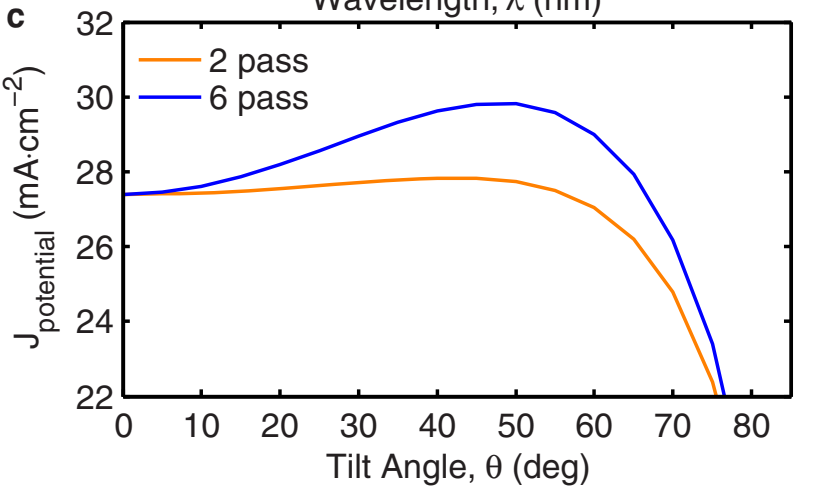

$3 \mid$ Absorption and external quantum efficiency of FLP devices. (a) Device (solid lines) and CQD film (dashed lines) absorption curves for flat (red) and FLP (green) samples. (b) External quantum efficiency of flat and FLP devices. $(\mathrm{b}$ - inset) Photograph of a tilted sample illuminated through the device aperture at $45^{\circ}$ with a thin strip of white light demonstrating the capturing of multiple passes of light - in this case the third and fifth passes can be clearly observed. For photovoltaic measurements, the light aperture defined the active area dimensions ${ }^{28}$. (c) Absorption and EQE enhancement of a FLP device as compared to a flat device. Internal quantum efficiency curves can be found in section SI 3.

light into the active layer is crucial to approaching complete absorption. It would be attractive to tailor the layers cladding the CQD material such that, following the first pass, light remained trapped within the CQD layer, wherein it would be waveguided and absorbed. In the absence of more strongly absorbing materials, folding the light path within the active layer of a solar cell remains a promising means to overcome the absorption versus extraction compromise.

\section{Methods}

Synthesis of $\mathrm{PbS}$ quantum dots and metal halide treatment. $\mathrm{PbS}$ quantum dots were synthesized according to a previously published method ${ }^{31}$. A solution-phase metal halide treatment $\left(\mathrm{CdCl}_{2}\right)$ was then carried out following a previously published method $^{8}$. Specifically, the metal halide precursor $\left(1 \mathrm{~mL}\right.$ of $\left.\mathrm{CdCl}_{2}\right)$ and

tetradecylphosphonic acid (TDPA) were dissolved in oleylamine with 13.6:1

Cd:TDPA molar ratio. This mixture was introduced into the CQD reaction flask after the sulfur source injection during the slow cooling process. A $6: 1 \mathrm{~Pb}: \mathrm{Cd}$ molar ratio was adopted during the synthesis. At $30-35^{\circ} \mathrm{C}$, the nanocrystals were isolated by the addition of $60 \mathrm{~mL}$ of acetone then subjected to centrifugation. The nanocrystals were then purified by dispersion in toluene and re-precipitation with a mixture of acetone/ 

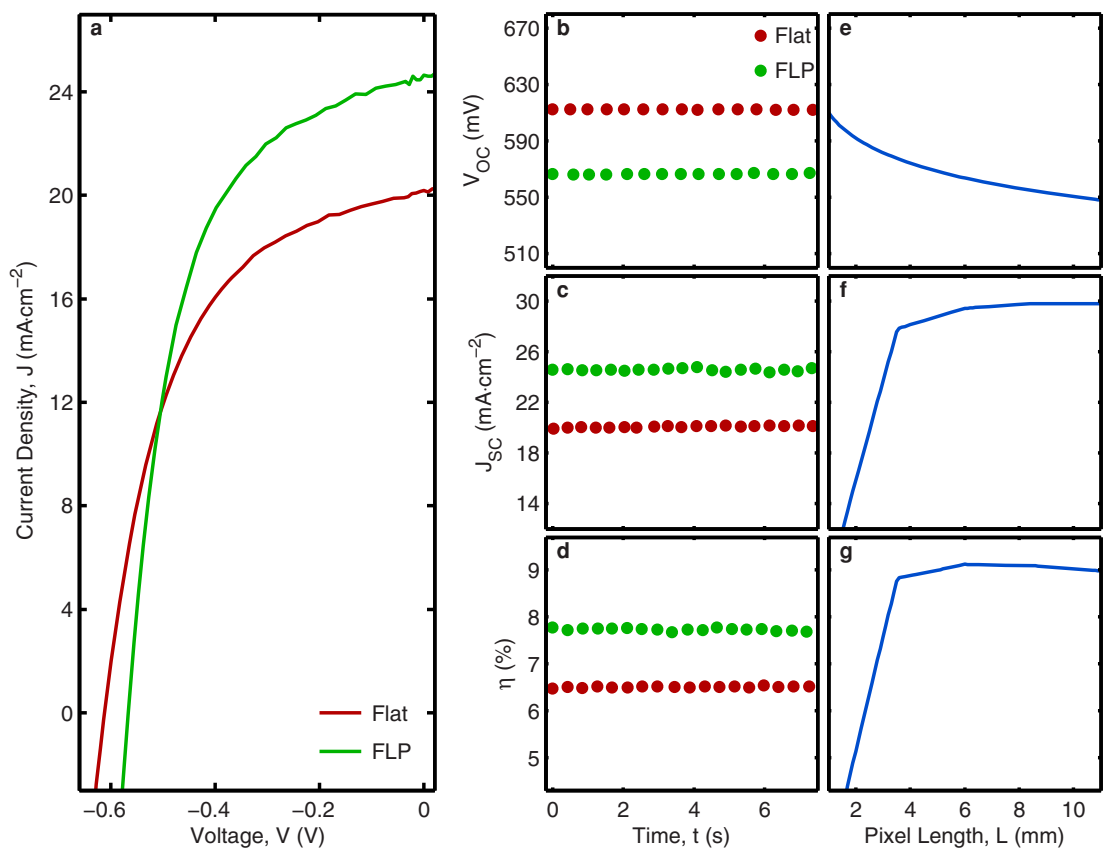

Figure $4 \mid$ Measured and theoretical photovoltaic device performance. (a) $J-V$ characteristics of flat and tilted samples illustrating a $\sim 4 \mathrm{~mA} \cdot \mathrm{cm}^{-2}$ enhancement in $J_{S C}$ and a $\sim 0.05 \mathrm{~V}$ reduction in $V_{O C}$. (b,c,d) Static $V_{O C}, J_{S C}$ and $\eta$ measurements, respectively, confirming a lack of rapid degradation and transient artefacts in flat and FLP device performance. (e,f,g) Theoretical implications of increased pixel strip length on $V_{O C}, J_{S C}$ and $\eta$.

methanol (1:1 volume ratio), then re-dissolved in anhydrous toluene. The solution was further washed with methanol two more times before finally dispersing it in octane at a concentration of $50 \mathrm{mg} / \mathrm{mL}$

Substrate preparation. Cleaned FTO-coated glass substrates (Pilkington, TEC 15) with a $45^{\circ}$ cut edge were employed in this study. We first deposited the edge mirror by e-beam evaporation of $5 \mathrm{~nm}$ of Ti at $1 \AA$ A/s followed by e-beam evaporation of $50 \mathrm{~nm}$ of Au at $1.5 \AA / \mathrm{s}$ followed by $100 \mathrm{~nm}$ of thermally evaporated Ag at $2 \AA / \mathrm{s}$. $50 \mathrm{~nm} \mathrm{TiO}$
(Kurt J. Lesker) was deposited to cover the substrate and its mirrored edge by RC magnetron sputtering at room temperature under Ar at 5 mTorr at a rate of $0.20 \AA / \mathrm{s}$. Both deposition systems were Angstrom Engineering Åmod deposition systems located in an Innovative Technology nitrogen filled glovebox. The substrates were then treated with a $120 \mathrm{~mm} \mathrm{TiCl}_{4}$ solution at $70^{\circ} \mathrm{C}$ for $30 \mathrm{~min}$ followed by a rinse with deionized water and annealing step on a hot plate at $520^{\circ} \mathrm{C}$ for $45 \mathrm{~min}$ in air ambient. The deposition of the bottom mirror was lastly done by e-beam evaporation of $50 \mathrm{~nm}$ of $\mathrm{Au}$ at $1.5 \AA / \mathrm{s}$ followed by $100 \mathrm{~nm}$ of thermally evaporated $\mathrm{Ag}$ at $2 \AA / \mathrm{s}$.

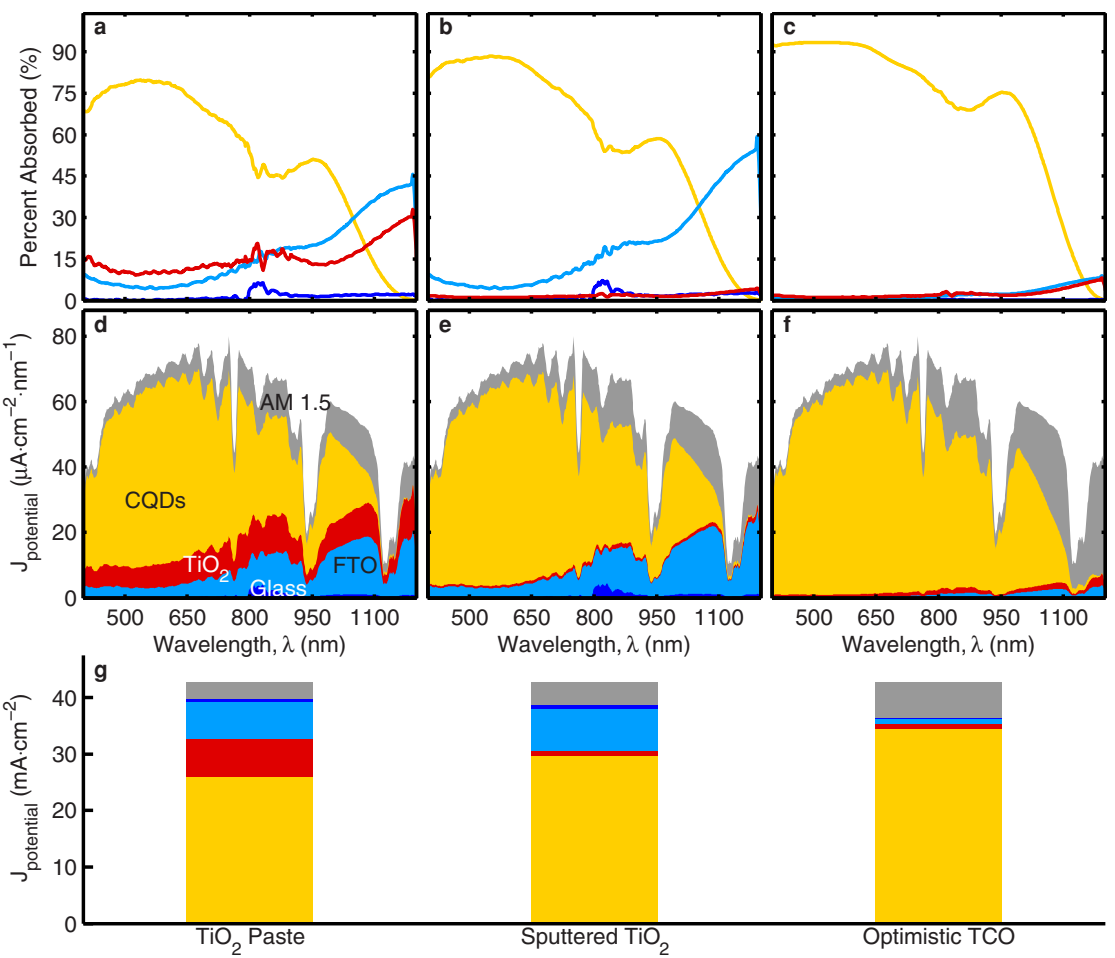

Figure $5 \mid$ Absorption contributions of constituent solar cell layers. (a,b,c) Percentage absorption contribution and (d,e,f) spectral $J_{S C}$ contribution of glass (navy), FTO (blue), $\mathrm{TiO}_{2}$ (red) and CQDs (yellow) for FLP devices employing $\mathrm{TiO}_{2}$ paste (a,d), sputtered $\mathrm{TiO}_{2}$ (b,e) and an optimistic substrate/ electrode combination $(\mathrm{c}, \mathrm{f})$. (g) Total absorbed photon equivalent $J_{S C}$ for all three cases. 
Device fabrication. PbS CQD films were deposited using a layer-by-layer spincoating process under an ambient atmosphere. For each layer, the CQD solution (50 $\mathrm{mg} / \mathrm{mL}$ in octane) was deposited on the substrate and spin-cast at $2500 \mathrm{RPM}$ for $10 \mathrm{~s}$. Solid-state ligand exchange was performed by flooding the surface with $1 \% \mathrm{v}: \mathrm{v}$ MPA in methanol for $3 \mathrm{~s}$ before spin-coating dry at $2500 \mathrm{RPM}$ for $10 \mathrm{~s}$. Two rinses with methanol were used to remove unbound ligands. Each device consisted of 10 layers. The top contacts were finally deposited and consisted of $10 \mathrm{~nm}$ thermally evaporated $\mathrm{MoO}_{3}$ deposited at a rate of $0.2 \AA / \mathrm{s}$, followed by e-beam deposition of $50 \mathrm{~nm}$ of Au deposited at $1.5 \AA / \mathrm{s}$, and finally $120 \mathrm{~nm}$ of thermally evaporated $\mathrm{Ag}$ deposited at $2.0 \AA / \mathrm{s}$.

AM 1.5 photovoltaic performance characterization. Current-voltage data were measured using a Keithley 2400 source meter. The solar spectrum at AM1.5 was simulated to within class A specifications (less than $25 \%$ spectral mismatch) with a xenon lamp and filters (ScienceTech; measured intensity of $100 \mathrm{~mW} \cdot \mathrm{cm}^{-2}$ ). The source intensity was measured with a Melles-Griot broadband power meter through a horizontal rectangular $0.02 \mathrm{~cm}^{2}$ aperture. We used an aperture slightly smaller than the top electrode to avoid overestimating the photocurrent: the entire photon fluence passing through the aperture was counted as incident on the device for all analyses of $\mathrm{J}_{\mathrm{SC}}$ and $\mathrm{EQE}^{28}$. The spectral mismatch of the system was characterized using a calibrated reference solar cell (Newport). The total AM 1.5 spectral mismatch - taking into account the simulator spectrum and the spectral responsivities of the test cell, reference cell, and broadband power meter - was re-measured periodically and found to be $\sim 11 \%$. This multiplicative factor, $\mathrm{M}=0.89$, was applied to the current density values of the J-V curve to most closely resemble true AM 1.5 performance ${ }^{32}$. The uncertainty of the current-voltage measurements was estimated to be $\pm 7 \%$.

EQE measurements. External quantum efficiency measurements were obtained by applying chopped $(220 \mathrm{kHz})$ monochromatic illumination (400 W xenon lamp through a monochromator with order-sorting filters) collimated and cofocused with a 1 Sun intensity white-light source on the device of interest. The power was measured with calibrated Newport 818-UV and Newport 818-IR power meters. The response from the chopped signal was measured using a Stanford Research Systems lock-in amplifier at short-circuit conditions. The uncertainty in the EQE measurements was estimated to be $\pm 8 \%$.

Absorption measurements. Absorption spectroscopy was carried out using a Cary 500 UV-vis-IR Scan photospectrometer with an attached integrating sphere. The FLP devices were angled at $45^{\circ}$ to mimic absorption in a tilted context.

1. O’Regan, B. \& Gratzel, M. A low-cost, high-efficiency solar cell based on dyesensitized colloidal $\mathrm{TiO}_{2}$ films. Nature 353, 737-740 (1991).

2. Shaheen, S. E. et al. $2.5 \%$ efficient organic plastic solar cells. Applied Physics Letters 78, 841-843 (2001)

3. Pattantyus-Abraham, A. G. et al. Depleted-Heterojunction Colloidal Quantum Dot Solar Cells. ACS Nano 4, 3374-3380 (2010).

4. Todorov, T. K., Reuter, K. B. \& Mitzi, D. B. High-Efficiency Solar Cell with EarthAbundant Liquid-Processed Absorber. Advanced Materials 22, E156-E159 (2010).

5. Luther, J. M. et al. Schottky Solar Cells Based on Colloidal Nanocrystal Films. Nano Lett. 8, 3488-3492 (2008).

6. Luther, J. M. et al. Stability Assessment on a 3\% Bilayer PbS/ZnO Quantum Dot Heterojunction Solar Cell. Advanced Materials 22, 3704-3707 (2010).

7. Ma, W. et al. Photovoltaic Performance of Ultrasmall PbSe Quantum Dots. ACS Nano 5, 8140-8147 (2011).

8. Ip, A. H. et al. Hybrid passivated colloidal quantum dot solids. Nature Nanotechnology 7, 577-582 (2012).

9. Kramer, I. J. \& Sargent, E. H. Colloidal Quantum Dot Photovoltaics: A Path Forward. ACS Nano 5, 8506-8514 (2011).

10. Zhitomirsky, D. et al. Colloidal Quantum Dot Photovoltaics: The Effect of Polydispersity. Nano Letters 12, 1007-1012 (2012).

11. Guyot-Sionnest, P. Electrical Transport in Colloidal Quantum Dot Films. J. Phys. Chem. Lett. 3, 1169-1175 (2012).

12. Leschkies, K. S., Jacobs, A. G., Norris, D. J. \& Aydil, E. S. Nanowire-quantum-dot solar cells and the influence of nanowire length on the charge collection efficiency. Applied Physics Letters 95, 193103-193103-3 (2009).

13. Barkhouse, D. A. R. et al. Depleted Bulk Heterojunction Colloidal Quantum Dot Photovoltaics. Advanced Materials 23, 3134-3138 (2011).

14. Kramer, I. J. et al. Ordered Nanopillar Structured Electrodes for Depleted Bulk Heterojunction Colloidal Quantum Dot Solar Cells. Advanced Materials 24, 2315-2319 (2012)
15. Lan, X. et al. Self-Assembled, Nanowire Network Electrodes for Depleted Bulk Heterojunction Solar Cells. Advanced Materials 25, 1769-1773 (2013).

16. Kramer, I. J., Levina, L., Debnath, R., Zhitomirsky, D. \& Sargent, E. H. Solar Cells Using Quantum Funnels. Nano Lett. 11, 3701-3706 (2011).

17. Brown, P. R. et al. Improved Current Extraction from $\mathrm{ZnO} / \mathrm{PbS}$ Quantum Dot Heterojunction Photovoltaics Using a $\mathrm{MoO}_{3}$ Interfacial Layer. Nano Lett. 11, 2955-2961 (2011).

18. Gao, J. et al. n-Type Transition Metal Oxide as a Hole Extraction Layer in PbS Quantum Dot Solar Cells. Nano Lett. 11, 3263-3266 (2011).

19. Paz-Soldan, D. et al. Jointly Tuned Plasmonic-Excitonic Photovoltaics Using Nanoshells. Nano Lett. 13, 1502-1508 (2013).

20. Choi, J. J. et al. Solution-Processed Nanocrystal Quantum Dot Tandem Solar Cells. Advanced Materials 23, 3144-3148 (2011).

21. Wang, X. et al. Tandem colloidal quantum dot solar cells employing a graded recombination layer. Nature Photonics 5, 480-484 (2011).

22. Sinton, R. A., Kwark, Y., Gan, J. Y. \& Swanson, R. M. 27.5-percent silicon concentrator solar cells. IEEE Electron Device Letters 7, 567-569 (1986).

23. Campbell, P. \& Green, M. A. Light trapping properties of pyramidally textured surfaces. Journal of Applied Physics 62, 243-249 (1987).

24. Heine, C. \& Morf, R. H. Submicrometer gratings for solar energy applications. Appl. Opt. 34, 2476-2482 (1995).

25. Rühle, S., Greenwald, S., Koren, E. \& Zaban, A. Optical Waveguide Enhanced Photovoltaics. Opt. Express 16, 21801-21806 (2008).

26. Standard Tables for Reference Solar Spectral Irradiances: Direct Normal and Hemispherical on $37^{\circ}$ Tilted Surface. (2008). doi:10.1520/G0173-03R08.

27. Koleilat, G. I. et al. A Donor-Supply Electrode (DSE) for Colloidal Quantum Dot Photovoltaics. Nano Lett. 11, 5173-5178 (2011).

28. Snaith, H. J. The perils of solar cell efficiency measurements. Nature Photonics 6 , 337-340 (2012)

29. Nelson, J. The Physics of Solar Cells. (Imperial College Press, 2003).

30. Henry, C. H. Limiting efficiencies of ideal single and multiple energy gap terrestrial solar cells. Journal of Applied Physics 51, 4494-4500 (1980).

31. Hines, M. A. \& Scholes, G. D. Colloidal PbS Nanocrystals with Size-Tunable NearInfrared Emission: Observation of Post-Synthesis Self-Narrowing of the Particle Size Distribution. Advanced Materials 15, 1844-1849 (2003).

32. Kroon, J. M., Wienk, M. M., Verhees, W. J. H. \& Hummelen, J. C. Accurate efficiency determination and stability studies of conjugated polymer/fullerene solar cells. Thin Solid Films 403-404, 223-228 (2002).

\section{Acknowledgements}

This publication is based on work in part supported by Award No. KUS-11-009-21, made by King Abdullah University of Science and Technology (KAUST). We thank Angstrom Engineering and Innovative Technologies for useful discussions regarding material deposition methods and control of glovebox environment, respectively. G.I.K acknowledges NSERC support in the form of Alexander Graham Bell Canada Graduate Scholarship. The authors acknowledge the International Cooperation of the Korea Institute of Energy Technology Evaluation and Planning (KETEP) grant funded by the Korea government Ministry of Knowledge Economy (2012T100100740). The authors would also like to acknowledge the technical assistance and scientific guidance of E. Palmiano, L. Levina, R. Wolowiec and D. Kopilovic.

\section{Author contributions}

G.I.K. and S.H. conceived of the project and undertook initial experiments. G.I.K., I.J.K. and C.T.O.W. designed, fabricated, characterized and optimized devices. A.J.L. and C.T.O.W. optimized the electrodes. I.J.K. developed the optical model. S.M.T. aided in the design of experiments project coordination. I.J.K. and E.H.S. wrote the manuscript. All authors edited and reviewed the manuscript.

\section{Additional information}

Supplementary information accompanies this paper at http://www.nature.com/ scientificreports

Competing financial interests: The authors declare no competing financial interests.

How to cite this article: Koleilat, G.I. et al. Folded-Light-Path Colloidal Quantum Dot Solar Cells. Sci. Rep. 3, 2166; DOI:10.1038/srep02166 (2013).

(c) (i) (2) This work is licensed under a Creative Commons Attribution-

C. visit http://creativecommons.org/licenses/by-nc-sa/3.0 